\title{
Short communication: Single molecule, real-time sequencing technology revealed species- and strain-specific methylation patterns of 2 Lactobacillus strains
}

\author{
Wenyi Zhang, Zhihong Sun, Bilige Menghe, and Heping Zhang ${ }^{1}$ \\ Key Laboratory of Dairy Biotechnology and Engineering, Education Ministry of P. R. China, Inner Mongolia Agricultural University, Hohhot, \\ Inner Mongolia 010018, P. R. China
}

\begin{abstract}
Pacific Biosciences' (Menlo Park, CA) single molecule, real-time sequencing technology was reported to have some advantages in generating finished genomes and characterizing the epigenome of bacteria. In the present study, this technology was used to sequence 2 Lactobacillus strains, Lactobacillus casei Zhang and Lactobacillus plantarum P-8. Previously, the former bacterium was sequenced by an Applied Biosystems 3730 DNA analyzer (Grand Island, NY), whereas the latter one was analyzed with Roche 454 (Indianapolis, IN) and Illumina sequencing technologies (San Diego, $\mathrm{CA}$ ). The results showed that single molecule, real-time sequencing resulted in high-quality, finished genomes for both strains. Interestingly, epigenome analysis indicates the presence of 1 active $\mathrm{N}^{6}$-methyladenine methyltransferase in $L$. casei Zhang, but none in L. plantarum P-8. Our study revealed for the first time a completely different methylation pattern in 2 Lactobacillus strains. Key words: SMRT sequencing technology, epigenome, base modification
\end{abstract}

\section{Short Communication}

Lactobacillus is a group of bacteria that can ferment hexose carbohydrates to produce predominantly lactic acid. The historical discovery and exploration of these bacteria are closely associated with food fermentations, where they remain important today with industrial application value (Stiles and Holzapfel, 1997). In addition to the indigenous food-related habitats of these bacteria (Zhang et al., 2008b; Fonseca et al., 2013; Nguyen et al., 2013), they are abundant in the gastrointestinal tracts of both humans and animals (Ahrné et al., 1998; Du Toit et al., 2001). Isolates from the same host species, including Lactobacillus plantarum and Lactobacillus casei, are often detected in a broad range of ecological

Received December 22, 2014.

Accepted January 26, 2015.

${ }^{1}$ Corresponding author: hepingdd@vip.sina.com. niches, reflecting their versatility (Kleerebezem et al., 2003; Zhang et al., 2010).

Genomics studies of Lactobacillus started with the publication of the L. plantarum WCSF1 in 2003 (Kleerebezem et al., 2003). Since then, benefitting from the quick development of DNA sequencing technology, the number of sequenced Lactobacillus genomes has grown exponentially. Currently, with the booming data from various genome-sequencing projects, we have unprecedented opportunities to gain insights into the genetic basis of Lactobacillus. Thus far, more than 182 genomes with abundant draft sequences from Lactobacillus are available, covering 54 different species (http://www. ncbi.nlm.nih.gov/). Although our understanding of Lactobacillus genetics has been significantly improved with the expanding information, their biological properties remain somewhat obscure due to the limited number of published complete genomes. Seemingly, new advances in cost-effective sequencing technologies that can help to decipher the sequenced genomes of Lactobacillus are still anticipated (Koren et al., 2012).

Pacific Biosciences' (Menlo Park, CA) single molecule, real-time sequencing technology (SMRT) was reported to have some advantages in assembling genomes and characterizing epigenome (Roberts et al., 2013). In particular, a lack of epigenomics study exists in Lactobacillus, which is of intense scientific interest. The existing evidence has shown that methylation does not only function in serving as part of a restrictionmodification (RM) system but also play important roles in chromosome stability, mismatch repair, replication, and gene expression (Low et al., 2001; Marinus and Casadesus, 2009). In the present study, SMRT was used to sequence $L$. casei Zhang and L. plantarum P-8 that were originally isolated from dairy products $(\mathrm{Wu}$ et al., 2009; Wang et al., 2014), and the newly generated genome data were further compared with the previous published genomes.

Lactobacillus casei Zhang and L. plantarum P-8 were obtained from the Collection Centre of Lactic Acid Bacteria at Inner Mongolia Agricultural University of China. The strains were grown in $5.0 \mathrm{~mL}$ of de Man, 
Table 1. General genome information of Lactobacillus casei Zhang and Lactobacillus plantarum P-8

\begin{tabular}{lcccccc}
\hline Strain & Plasmid & Size (bp) & GC content $(\%)$ & rRNA & transfer RNA & CDS $^{1}$ \\
\hline L. casei Zhang & 1 & $2,861,969$ & 46.51 & 15 & 59 & 2,807 \\
L. plantarum P-8 & 7 & $3,035,719$ & 44.8 & 16 & 67 & 2,952 \\
\hline${ }^{1}$ CDS $=$ coding sequence & & & & &
\end{tabular}

${ }^{1} \mathrm{CDS}=$ coding sequence.

Rogosa, Sharpe broth (Oxoid, Hampshire, UK) at $37^{\circ} \mathrm{C}$ for 18 to $22 \mathrm{~h}$. The DNA isolation was performed using the Wizard Genomic DNA Purification Kit (Promega, Madison, WI). The integrity of DNA was examined by $0.6 \%$ agarose gel and $1.2 \%$ lonza gel electrophoresis. The SMRT bell template library preparation was performed as described previously (Eid et al., 2009; Korlach et al., 2010).

The SMRT sequencing was performed on a PacBio RS II instrument (Pacific Biosciences). De novo assemblies were subjected to standard hierarchical genome assembly process (Chin et al., 2013) using just PacBio data from a single, long-insert library, and the consensus was called across reads after assembly polishing. Polymorphic sites were identified with Quiver algorithm available in the Pacific Biosciences SMRT Portal (version 2.3.0). Interpulse durations were measured as previously described (Murray et al., 2012). To identify the common base modifications and analyze methyltransferase motifs, the protocol for modification and motif analysis in the software was selected, which used an in silico kinetic reference and a $t$-test-based kinetic score detection of modified base positions. Visualization of the information from the data analysis was displayed in SMRT View.

Gene prediction was performed by using Glimmer and annotated using BLAST against nonredundant database (provided by the National Center for Biotechnology Information; Altschul et al., 1990; Delcher et al., 2007). The transfer RNA were predicted using tRNAscan-SE (Lowe and Eddy, 1997). The rRNA were predicted using RNAmmer (Lagesen et al., 2007). Function category was predicted with clusters of orthologous genes and Kyoto Encyclopedia of Genes and Genomes databases (Tatusov et al., 1997). Restrictionmodification systems were identified by scanning locally for homologs in REBASE (Roberts et al., 2010). The characterization of protein family, domain, and biologically significant sites was carried out using Interproscan (Hunter et al., 2012). The accession numbers for the chromosomes of $L$. casei Zhang and L. plantarum P-8 were CP001084 and CP005942, and were CP000935 (plca36), CP005943-CP005948 (LBPp1-LBPp6), and CP010527 (LBPp7) for the plasmids.

Previously, L. casei Zhang was sequenced by an Applied Biosystems 3730 DNA analyzer (Grand Island,
NY), whereas L. plantarum P-8 was analyzed with Roche 454 (Indianapolis, IN) and Illumina sequencing technologies (San Diego, CA). The 2 strains bear 1 and 6 extra chromosomal plasmids, correspondingly (Zhang et al., 2008a, 2010, 2015). Compared with the 2 reference genomes, SMRT sequencing resulted in higher quality, finished genomes for both strains. The de novo assemblies were fully collinear with the reference genomes. The general information of the 2 genomes was described in Table 1.

The hierarchical genome assembly process assembled genome of $L$. casei Zhang resulted in 2 contigs. A total of 137 SNP were identified, which differed from the reference. In L. plantarum $\mathrm{P}-8,51 \mathrm{SNP}$ and 10 insertions were detected compared with the chromosome reference. The insertion sequences were collapsed repeats uniquely resolved de novo by PacBio, which were missing in the reference. Three of the 7 plasmids were significantly different from the reference sequences achieved from the public DNA databases. The PacBio reads have provided direct evidence that the reference sequences of these 3 plasmids were not correct. Specifically, numerous breaks and areas of poor coverage were found in the shotgun read structure of the plasmid LBPp4. The plasmid LBPp7 was uniquely resolved de novo by PacBio, which was missing in the original reference sequence. Moreover, regional similarity with the reference plasmid of LBPp1 could be identified. Collectively, repeat elements common to both plasmids and chromosomes likely caused the errors in the reference.

In recent years, the next-generation sequencing technologies including the shotgun methods have been commonly used to sequence bacterial genomes. However, the frequency of repeated sequences in the bacterial genomes causes complications and difficulties in the genome assembly process. Our data have clearly indicated that the long reads generated by the SMRT instrument overcome the difficulties brought about by these repeats. Similar successes were reported by Chin et al. (2013), that assembly using only SMRT reads achieved comparable performance relative to other DNA sequencing platforms.

To identify methylated sites within the genomes of L. casei Zhang and L. plantarum P-8, 2 SMRT cells for each strain were run using libraries with a cutoff of $7 \mathrm{~kb}$ and the adoption of XL polymerase enzyme 
(a)

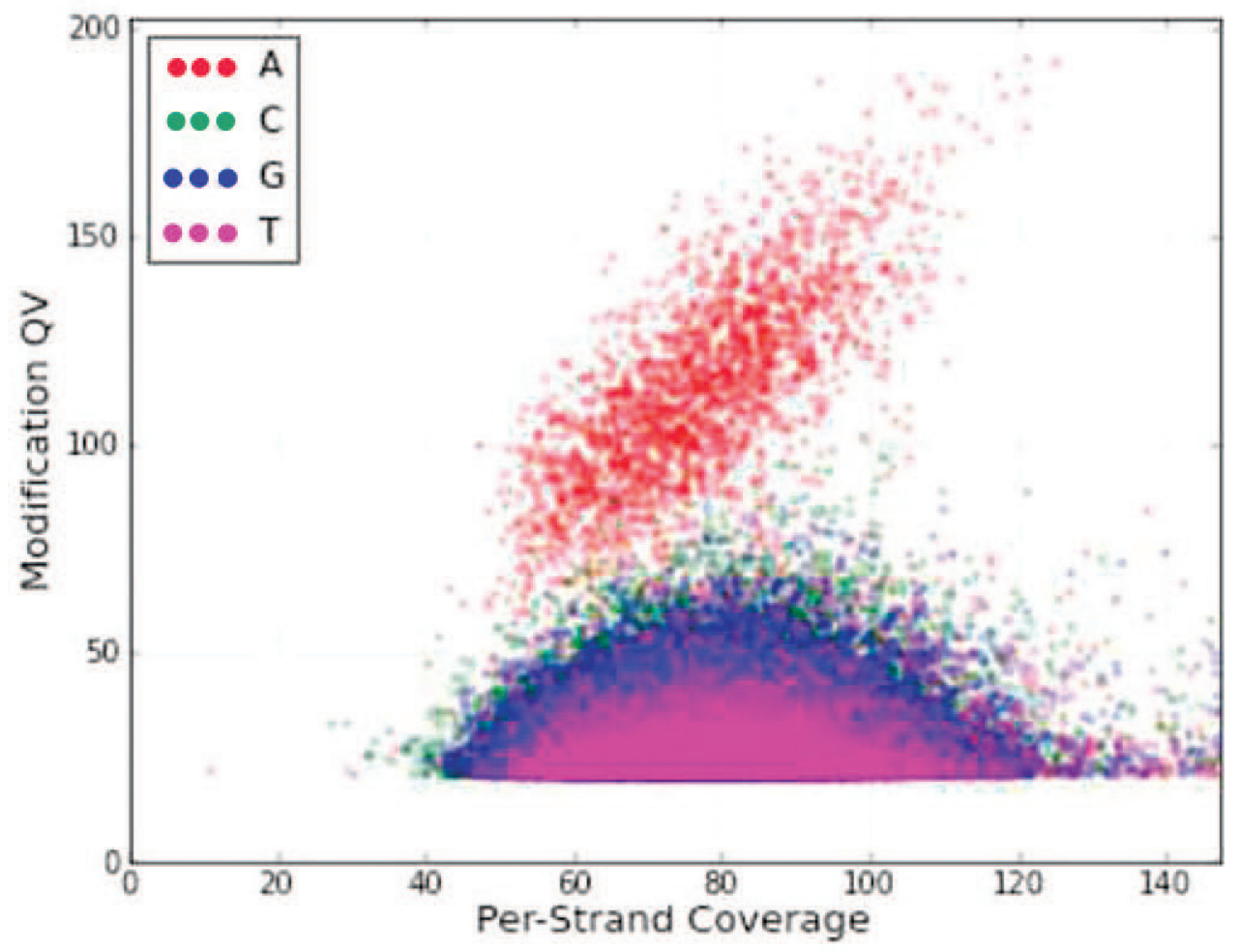

(b)

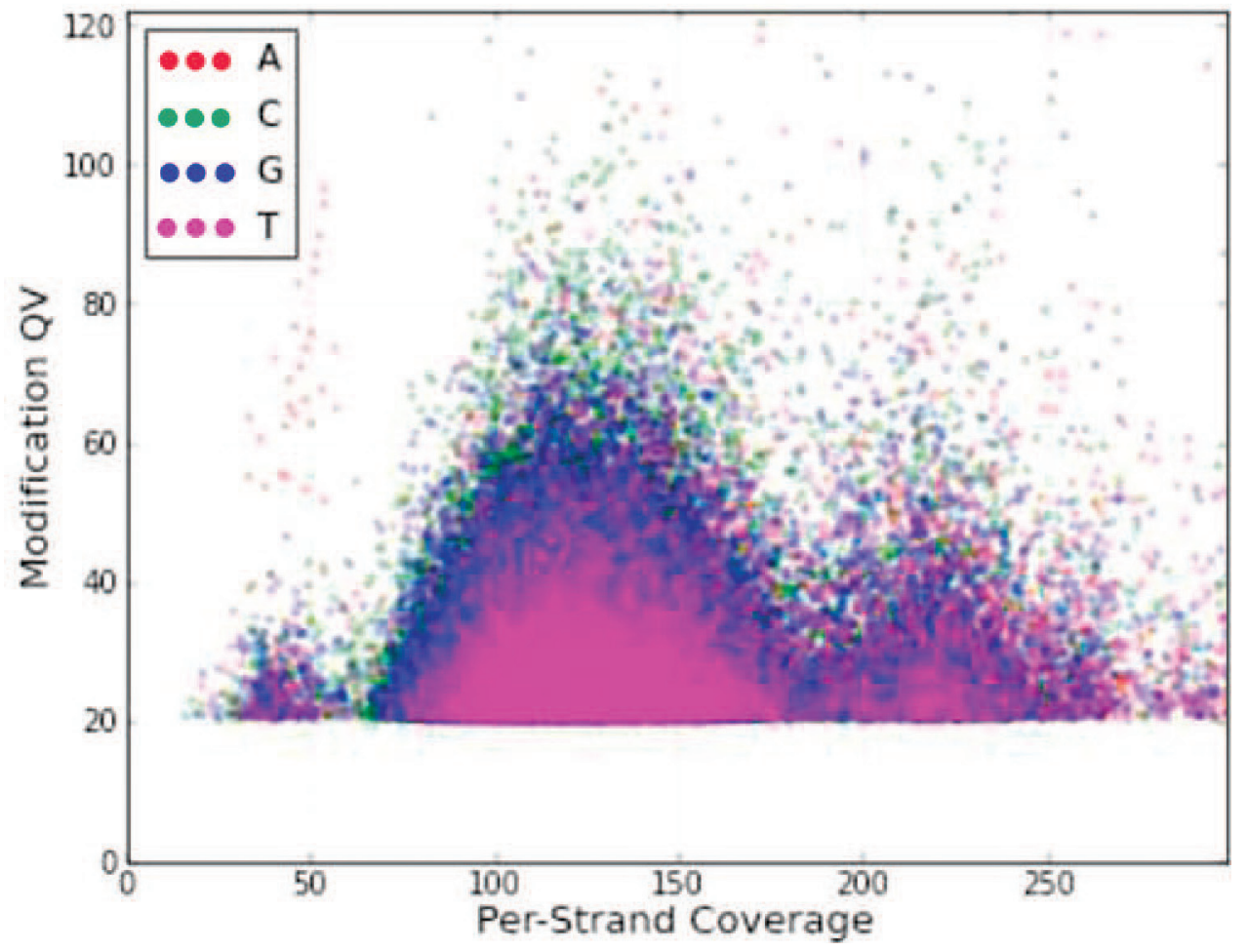

Figure 1. Scatter plot of sequencing coverage and modification quality value (QV) in Lactobacillus casei Zhang (a) and Lactobacillus plantarum P-8 (b). 
(Pacific Biosciences), during which a 180-min movie was captured. Potential methyltransferases that were responsible for the methylation were predicted using REBASE. According to the standard recommendation from SMRT for detecting the common base modifications, sequence data obtained should have an average coverage of $>100 \times$ (which was $148.31 \times$ for $L$. casei Zhang and $224.49 \times$ for L. plantarum P-8).

Bioinformatics inspection of the genomes revealed the presence of 2 putative type II RM system-associated methyltransferases (LCAZH_2054 and LCAZH_2056) in L. casei Zhang. Both of them were found to have a typical signature of N6 adenine-specific DNA methyltransferase that belongs to the N12 class (IPR002296). Interestingly, the protein coded by LCAZH_2054 contained 1 additional site named DNA methylase, N6 adenine-specific, conserved site (IPR002052), which was suggestive of catalytic activity. Consistent with these predictions, 1 active $\mathrm{N}^{6}$-methyladenine methyltransferase was identified in L. casei Zhang by SMRT sequencing (Figure 1a). Further genome-wide motif analysis via SMRT Portal identified the $5^{\prime}$-ACRC ${ }^{\mathrm{m} 6} \mathrm{AG}-3^{\prime}$ as the 6-methyladenine (6-mA) recognition sequences. Based on the presence of $5^{\prime}$-ACRC ${ }^{\mathrm{m} 6} \mathrm{AG}-3^{\prime}$ signature, 1,906 methylation sites were predicted in the genome, accounting for $>99.8 \%$ of all hits matching such motifs. In L. plantarum P-8, a total of 9 loci (LBP_cg0731, LBP_cg0733, LBP_cg0734, LBP_cg0736, LBP cg0738, LBP_cg1666, LBP_p4g027, LBP_p4g028, and LBP_p4g029) were predicted to be the components of type I and type II RM systems. However, no active methyltransferase was found by SMRT analysis. As shown in Figure 1b, only background signals, but no population of separated kinetic scores, were detected.

It is known that the DNA of most organisms comprises more than just the 4 canonical bases (A, C, G, and $\mathrm{T}$ ). Apart from mammals, many bacterial genomes also contain a variety of modified DNA bases, including the methylated ones. The regulation of gene expression by DNA base modification, such as DNA methylation, is not limited in mammals but also widely found in bacterial genomes. The most common forms of methylation are 6-mA, 4-methylcytosine, and 5-methylcytosine (Clark et al., 2013). For example, 5-methylcytosine methylation is a regulator of the stationary phase gene expression in Escherichia coli, as identified by bisulfate conversion (Kahramanoglou et al., 2012). By using SMRT, Murray et al. (2012) reported the discoveries of new 6-mA and 4-methylcytosine methylation patterns in Geobacter metallireducens, Chromohalobacter salexigens, Vibrio breoganii, Bacillus cereus, Campylobacter jejuni ssp. jejuni and C. jejuni, and the respective DNA methyltransferases responsible for the methylation patterns. In the genus Lactobacillus, the presence of methylation has not been reported yet. Herein, we have exclusively found $6-\mathrm{mA}$ in $L$. casei but not $L$. plantarum, although whether such distribution pattern in these 2 studied strains is representative at the species level needs to be further explored. Finally, our current observations have opened an interesting area for future study concerning whether and how bacterial genome methylation is related to its physiological functioning, particularly for each specific species or strain.

In the present study, we demonstrated that SMRT is able to provide highly accurate genomics data of 2 Lactobacillus strains. Meanwhile, we generated preliminary data on the base modification in 2 Lactobacillus strains, which further revealed a possible species- or strain-specific methylation pattern. As little is known about the epigenetics of this genus, the utilization of such technologies may open a new window for understanding its biology and function.

\section{ACKNOWLEDGMENTS}

This research was supported by the National Natural Science Foundation of China (Beijing; Grant No. 31430066 and 31201396), Hi-Tech Research and Development Program of China (Beijing; 863 Planning, Grant No. 2011AA100901 and 2011AA100902), International S \& T Cooperation Program of China (Beijing; ISTCP, Grant No. 2014DFR31150), and the China Agriculture Research System (Beijing; Grant No. CARS-37). We thank Kwok Lai Yu from our research group for her critical reading of the manuscript, and Hanqian $\mathrm{Zu}$, Yue Chen, and Dan Li from Gene Company Limited in China for their technical support.

\section{REFERENCES}

Ahrné, S., S. Nobaek, B. Jeppsson, I. Adlerberth, A. E. Wold, and G. Molin. 1998. The normal Lactobacillus flora of healthy human rectal and oral mucosa. J. Appl. Microbiol. 85:88-94.

Altschul, S. F., W. Gish, W. Miller, E. W. Myers, and D. J. Lipman. 1990. Basic local alignment search tool. J. Mol. Biol. 215:403-410.

Chin, C. S., D. H. Alexander, P. Marks, A. A. Klammer, J. Drake, C. Heiner, A. Clum, A. Copeland, J. Huddleston, E. E. Eichler, S. W. Turner, and J. Korlach. 2013. Nonhybrid, finished microbial genome assemblies from long-read SMRT sequencing data. Nat. Methods 10:563-569.

Clark, T. A., X. Lu, K. Luong, Q. Dai, M. Boitano, S. W. Turner, C. He, and J. Korlach. 2013. Enhanced 5-methylcytosine detection in single-molecule, real-time sequencing via Tet1 oxidation. BMC Biol. 11:4.

Delcher, A. L., K. A. Bratke, E. C. Powers, and S. L. Salzberg. 2007. Identifying bacterial genes and endosymbiont DNA with Glimmer. Bioinformatics 23:673-679.

Du Toit, M., L. M. Dicks, and W. H. Holzapfel. 2001. Taxonomy of obligately homofermentative and facultatively heterofermentative lactobacilli in pig faeces. Lett. Appl. Microbiol. 32:199-204.

Eid, J., A. Fehr, J. Gray, K. Luong, J. Lyle, G. Otto, P. Peluso, D. Rank, P. Baybayan, B. Bettman, A. Bibillo, K. Bjornson, B. Chaudhuri, F. Christians, R. Cicero, S. Clark, R. Dalal, A. Dewinter, J. Dixon, M. Foquet, A. Gaertner, P. Hardenbol, C. Heiner, K. 
Hester, D. Holden, G. Kearns, X. Kong, R. Kuse, Y. Lacroix, S. Lin, P. Lundquist, C. Ma, P. Marks, M. Maxham, D. Murphy, I. Park, T. Pham, M. Phillips, J. Roy, R. Sebra, G. Shen, J. Sorenson, A. Tomaney, K. Travers, M. Trulson, J. Vieceli, J. Wegener, D. Wu, A. Yang, D. Zaccarin, P. Zhao, F. Zhong, J. Korlach, and S. Turner. 2009. Real-time DNA sequencing from single polymerase molecules. Science 323:133-138.

Fonseca, S., A. Cachaldora, M. Gomez, I. Franco, and J. Carballo. 2013. Monitoring the bacterial population dynamics during the ripening of Galician chorizo, a traditional dry fermented Spanish sausage. Food Microbiol. 33:77-84.

Hunter, S., P. Jones, A. Mitchell, R. Apweiler, T. K. Attwood, A. Bateman, T. Bernard, D. Binns, P. Bork, S. Burge, E. de Castro, P. Coggill, M. Corbett, U. Das, L. Daugherty, L. Duquenne, R D. Finn, M. Fraser, J. Gough, D. Haft, N. Hulo, D. Kahn, E. Kelly, I. Letunic, D. Lonsdale, R. Lopez, M. Madera, J. Maslen, C. McAnulla, J. McDowall, C. McMenamin, H. Mi, P. MutowoMuellenet, N. Mulder, D. Natale, C. Orengo, S. Pesseat, M. Punta, A. F. Quinn, C. Rivoire, A. Sangrador-Vegas, J. D. Selengut, C. J. Sigrist, M. Scheremetjew, J. Tate, M. Thimmajanarthanan, P. D. Thomas, C. H. Wu, C. Yeats, and S. Y. Yong. 2012. InterPro in 2011: New developments in the family and domain prediction database. Nucleic Acids Res. 40:D306-D312.

Kahramanoglou, C., A. I. Prieto, S. Khedkar, B. Haase, A. Gupta, V. Benes, G. M. Fraser, N. M. Luscombe, and A. S. Seshasayee. 2012. Genomics of DNA cytosine methylation in Escherichia coli reveals its role in stationary phase transcription. Nat. Commun. 3:886.

Kleerebezem, M., J. Boekhorst, R. van Kranenburg, D. Molenaar, O. P. Kuipers, R. Leer, R. Tarchini, S. A. Peters, H. M. Sandbrink, M. W. Fiers, W. Stiekema, R. M. Lankhorst, P. A. Bron, S. M. Hoffer, M. N. Groot, R. Kerkhoven, M. de Vries, B. Ursing, W M. de Vos, and R. J. Siezen. 2003. Complete genome sequence of Lactobacillus plantarum WCFS1. Proc. Natl. Acad. Sci. USA 100:1990-1995.

Koren, S., M. C. Schatz, B. P. Walenz, J. Martin, J. T. Howard, G. Ganapathy, Z. Wang, D. A. Rasko, W. R. McCombie, E. D. Jarvis, and M. P. Adam. 2012. Hybrid error correction and de novo assembly of single-molecule sequencing reads. Nat. Biotechnol. 30:693-700.

Korlach, J., K. P. Bjornson, B. P. Chaudhuri, R. L. Cicero, B. A. Flusberg, J. J. Gray, D. Holden, R. Saxena, J. Wegener, and S. W. Turner. 2010. Real-time DNA sequencing from single polymerase molecules. Methods Enzymol. 472:431-455.

Lagesen, K., P. Hallin, E. A. Rodland, H. H. Staerfeldt, T. Rognes, and D. W. Ussery. 2007. RNAmmer: Consistent and rapid annotation of ribosomal RNA genes. Nucleic Acids Res. 35:3100-3108.

Low, D. A., N. J. Weyand, and M. J. Mahan. 2001. Roles of DNA adenine methylation in regulating bacterial gene expression and virulence. Infect. Immun. 69:7197-7204.
Lowe, T. M., and S. R. Eddy. 1997. tRNAscan-SE: A program for improved detection of transfer RNA genes in genomic sequence. Nucleic Acids Res. 25:955-964.

Marinus, M. G., and J. Casadesus. 2009. Roles of DNA adenine methylation in host-pathogen interactions: Mismatch repair, transcriptional regulation, and more. FEMS Microbiol. Rev. 33:488-503.

Murray, I. A., T. A. Clark, R. D. Morgan, M. Boitano, B. P. Anton, K. Luong, A. Fomenkov, S. W. Turner, J. Korlach, and R. J. Roberts. 2012. The methylomes of six bacteria. Nucleic Acids Res. 40:11450-11462.

Nguyen, D. T., K. Van Hoorde, M. Cnockaert, E. De Brandt, M. Aerts, L. Binh Thanh, and P. Vandamme. 2013. A description of the lactic acid bacteria microbiota associated with the production of traditional fermented vegetables in Vietnam. Int. J. Food Microbiol. 163:19-27.

Roberts, R. J., M. O. Carneiro, and M. C. Schatz. 2013. The advantages of SMRT sequencing. Genome Biol. 14:405.

Roberts, R. J., T. Vincze, J. Posfai, and D. Macelis. 2010. REBASEA database for DNA restriction and modification: Enzymes, genes and genomes. Nucleic Acids Res. 38:D234-D236.

Stiles, M. E., and W. H. Holzapfel. 1997. Lactic acid bacteria of foods and their current taxonomy. Int. J. Food Microbiol. 36:1-29.

Tatusov, R. L., E. V. Koonin, and D. J. Lipman. 1997. A genomic perspective on protein families. Science 278:631-637.

Wang, L., J. Zhang, Z. Guo, L. Kwok, C. Ma, W. Zhang, Q. Lv, W. Huang, and H. Zhang. 2014. Effect of oral consumption of probiotic Lactobacillus planatarum P-8 on fecal microbiota, SIgA, SCFAs, and TBAs of adults of different ages. Nutrition 30:776-783.

Wu, R. L. Wang, J. Wang, H. Li, B. Menghe, J. Wu, M. Guo, and H. Zhang. 2009. Isolation and preliminary probiotic selection of lactobacilli from koumiss in Inner Mongolia. J. Basic Microbiol. 49:318-326.

Zhang, W., Z. Sun, M. Bilige, and H. Zhang. 2015. Complete genome sequence of probiotic Lactobacillus plantarum P-8 with antibacterial activity. J. Biotechnol. 193:41-42.

Zhang, W., D. Yu, Z. Sun, X. Chen, Q. Bao, H. Meng, S. Hu, and H. Zhang. 2008a. Complete nucleotide sequence of plasmid plca36 isolated from Lactobacillus casei Zhang. Plasmid 60:131-135.

Zhang, W., D. Yu, Z. Sun, R. Wu, X. Chen, W. Chen, H. Meng, S. $\mathrm{Hu}$, and H. Zhang. 2010. Complete genome sequence of Lactobacillus casei Zhang, a new probiotic strain isolated from traditional homemade koumiss in Inner Mongolia, China. J. Bacteriol. 192:5268-5269.

Zhang, W. Y., Y. Y. Yun, T. S. Sun, B. Menghe, and H. P. Zhang. 2008b. Isolation and identification of dominant microorganisms involved in naturally fermented goat milk in Haixi region of Qinghai, China. Ann. Microbiol. 58:213-217. 